\title{
Smoking Abstinence-Induced Changes in Resting State Functional Connectivity with Ventral Striatum Predict Lapse During a Quit Attempt
}

\author{
Maggie M Sweitzer*, ', Charles F Geier², Merideth A Addicott', Rachel Denlinger ${ }^{3}$, Bethany R Raiff', \\ Jesse Dallery ${ }^{5}$, F Joseph McClernon' and Eric C Donny ${ }^{3,6,7,8}$ \\ 'Department of Psychiatry and Behavioral Sciences, Duke University Medical Center, Durham, NC, USA; ${ }^{2}$ Department of Human Development \\ and Family Studies, Penn State University, State College, PA, USA; ${ }^{3}$ Department of Behavioral and Social Sciences, Brown University, Providence, RI, \\ USA; ${ }^{4}$ Department of Psychology, Rowan University, Glassboro, NJ, USA; ${ }^{5}$ Department of Psychology, University of Florida, Gainesville, FL, USA; \\ ${ }^{6}$ Department of Psychology, University of Pittsburgh, Pittsburgh, PA, USA; ' Department of Psychiatry, University of Pittsburgh Medical Center, \\ Pittsburgh, PA, USA; ${ }^{8}$ Center for Neural Basis of Cognition, University of Pittsburgh and Carnegie Mellon University, Pittsburgh, PA, USA
}

\begin{abstract}
The ventral and dorsal striatum are critical substrates of reward processing and motivation and have been repeatedly linked to addictive disorders, including nicotine dependence. However, little is known about how functional connectivity between these and other brain regions is modulated by smoking withdrawal and may contribute to relapse vulnerability. In the present study, 37 smokers completed resting state $\mathrm{fMRI}$ scans during both satiated and 24-h abstinent conditions, prior to engaging in a 3-week quit attempt supported by contingency management. We examined the effects of abstinence condition and smoking outcome (lapse vs non-lapse) on whole-brain connectivity with ventral and dorsal striatum seed regions. Results indicated a significant condition by lapse outcome interaction for both right and left ventral striatum seeds. Robust abstinence-induced increases in connectivity with bilateral ventral striatum were observed across a network of regions implicated in addictive disorders, including insula, superior temporal gyrus, and anterior/mid-cingulate cortex among non-lapsers; the opposite pattern was observed for those who later lapsed. For dorsal striatum seeds, 24-h abstinence decreased connectivity across both groups with several regions, including medial prefrontal cortex, posterior cingulate cortex, hippocampus, and supplemental motor area. These findings suggest that modulation of striatal connectivity with the cingulo-insular network during early withdrawal may be associated with smoking cessation outcomes.
\end{abstract}

Neuropsychopharmacology (20 I6) 4I, 252 I-2529; doi: I 0. I 038/npp.20 I6.56; published online II May 20 I6

\section{INTRODUCTION}

Multiple brain regions are implicated in addictive disorders, including tobacco dependence. In particular, the ventral and dorsal striatum (VS and DS) - key nodes in a larger functional network supporting reward valuation, incentive salience, and motivated behavior-play a critical role in drug reinforcement (Nestler, 2004) and the transition to compulsive drug use (Everitt and Robbins, 2005), respectively. Drugs of abuse, including nicotine, act to increase extracellular dopamine within the striatum, and with repeated exposure are thought to 'hijack' natural reward circuitry by increasing drive to obtain the drug (Volkow et al, 2009). Neuroimaging studies of human smokers have consistently demonstrated heightened blood oxygenation level dependent (BOLD)

*Correspondence: Dr MM Sweitzer, Department of Psychiatry and Behavioral Sciences, Duke University Medical Center, 2608 Erwin Rd, Suite 300, Durham, NC 277I0, USA, Tel: + I 919668 0094, Fax: + I 91968 I 0016, E-mail: maggie.sweitzer@duke.edu

Received 8 November 2015; revised 19 March 2016; accepted 12 April 2016; accepted article preview online 19 April 2016 response to smoking stimuli relative to neutral stimuli in the striatum and other interconnected brain regions (Buhler et al, 2010; David et al, 2005; Engelmann et al, 2012; McClernon et al, 2009; Sweitzer et al, 2013). Furthermore, alterations in striatal processing of smoking and other rewarding stimuli have been associated with increased craving (Sweitzer et al, 2013) and predict smoking behavior (Versace et al, 2014; Wilson et al, 2014).

Despite the critical role of the striatum in addictive disorders, including nicotine dependence, less is known about how alterations in circuit-level function contribute to smoking behavior and relapse vulnerability. The VS and DS are heavily interconnected with multiple cortical and subcortical regions, including the orbitofrontal and ventromedial prefrontal cortex, anterior cingulate cortex, amygdala, thalamus, and anterior insula (Middleton and Strick, 2002; Reynolds and Zahm, 2005; Wright and Groenewegen, 1996). A growing literature has identified altered patterns of resting state functional connectivity (rsFC) within mesocorticolimbic circuitry among drug-dependent individuals, including smokers, relative to healthy controls (Sutherland et al, 
2012). Among smokers, reduced rsFC strength between the striatum and dorsal anterior cingulate (dACC) is associated with greater nicotine dependence severity (Hong et al, 2009, 2010), and DS activation to smoking stimuli is positively correlated with rsFC strength across regions of the prefrontal and parietal cortex (Janes et al, 2012). In another study, enhanced connectivity during a smoking cuereactivity task within a network including the striatum, dACC and insula, predicted decreased likelihood of lapse (Janes et al, 2010).

Together, these findings suggest that variability in striatal connectivity with cognitive control regions has important implications for smoking behavior. However, little is known about how striatal connectivity is modulated by withdrawal or may impact cessation success. The impact of withdrawal on brain function is particularly important given that most smokers relapse during early stages of a quit attempt, when withdrawal is heightened (Hughes, 2007). Recent evidence suggests that connectivity between large-scale brain networks is altered by abstinence from smoking (Cole et al, 2010; Lerman et al, 2014; Wang et al, 2014); however, these studies examined changes in global brain connectivity or large-scale networks that did not explicitly include the striatum, so the extent to which abstinence impacts striatal connectivity with other brain regions is unknown. One study using a seed-based approach to examine rsFC between the dACC and striatum found no effect of nicotine administration on connectivity between these regions; however, effects of withdrawal were not examined (Hong et al, 2009). Furthermore, to our knowledge, no study has prospectively investigated associations between abstinence-induced changes in rsFC and the ability to abstain from smoking during a quit attempt.

The present study examined (a) the effects of 24-h smoking abstinence (compared with satiety) on rsFC using a striatal seed-based connectivity analysis; and (b) whether abstinence-induced changes in rsFC differed among smokers who subsequently maintained abstinence compared with those who lapsed during a 3-week quit attempt supported by contingency management (CM). Based on prior studies implicating attenuated connectivity with the dACC and insula in nicotine dependence and relapse (Hong et al, 2010; Janes et al, 2010), we hypothesized that 24-h abstinence might further decrease rsFC strength between striatal seeds and these regions, and that smokers who lapsed during $\mathrm{CM}$ would exhibit weaker rsFC during 24-h abstinence relative to satiety.

\section{MATERIALS AND METHODS}

\section{Participants}

Participants were eligible for study inclusion if they were between 18 and 65 years old, reported smoking $\geqslant 5$ cigarettes per day during the past year, had expired carbon monoxide (CO) level of $\geqslant 8 \mathrm{ppm}$, and were willing to make a quit attempt. Exclusion criteria included self-reported psychiatric illness or significant medical illness in the past year; reported history of serious traumatic brain injury; current heavy drug or alcohol use determined by self-report (drug use for $10+$ days or 4 or more drinks per day for $10+$ days in past 30 ); positive urine drug screen or breath alcohol $>0.08$; current use of any psychotropic medication or other tobacco products; interest in using smoking cessation medications; pregnancy/lactation; head injury with loss of consciousness in the past year; claustrophobia; and any known risk from exposure to high-field strength magnetic fields. Because the parent study involved a genetic component, participation was restricted to Caucasians to minimize population stratification. All participants provided informed consent, and study procedures were approved by the University of Pittsburgh Institutional Review Board.

Out of 56 individuals initially recruited, 12 withdrew from the study prior to completion of fMRI sessions and 7 were excluded from analyses due to technical issues (see Supplementary Information). Participants included in analyses $(n=37)$ had a mean age of $33.6( \pm 12.5)$, had a mean score of $3.5( \pm 2.4)$ on the Fagerström Test for Nicotine Dependence (FTND) (Heatherton et al, 1991), and smoked an average of 13.6 cigarettes per day $( \pm 6.1)$ for the past $14.0( \pm 11.1)$ years; $51 \%$ were female. Only two participants endorsed illicit drug use in the past 30 days (marijuana and hallucinogens, on two occasions).

\section{Procedure}

Screening session. Eligible participants attended an inperson screening. Breath and urine samples were collected to assess breath alcohol level, illicit drug use, and pregnancy. Expired $\mathrm{CO}$ was assessed upon arrival and after smoking a cigarette in the laboratory. To prevent exclusion of participants who had not smoked recently prior to the visit, the minimum $\mathrm{CO}$ inclusion criterion was satisfied if either CO sample was greater than $8 \mathrm{ppm}$. Participants completed questionnaires assessing demographics, medical and psychiatric history, nicotine use history, and nicotine dependence (FTND).

fMRI sessions. Participants completed two identical fMRI sessions on separate days (mean of 16.6 days apart), once following smoking ad libitum ('satiated') and once after 24-h abstinence ('abstinent'). Session order was randomly assigned; 16 participants (43\%) completed the abstinent session first. Abstinence was verified via self-report and expired $\mathrm{CO}<8 \mathrm{ppm}$ or $50 \%$ reduction from baseline. Prior to each scan participants underwent task training and completed subjective measures, including a 4 -item version of the Questionnaire on Smoking Urges (QSU-4; Toll et al, 2006) and the Minnesota Nicotine Withdrawal Scale (MNWS; Hughes and Hatsukami, 1986). The QSU-4 was repeated immediately after the scans, which lasted $1 \mathrm{~h}$. During the satiated session, participants smoked a cigarette immediately prior to the scan. During each fMRI scan participants completed a rewarded guessing task (reported separately; Sweitzer et al, 2016) followed by a 7-min resting scan, during which they were instructed to close their eyes, relax, and remain still.

Contingency management (CM). Following completion of both fMRI sessions, participants made a smoking quit attempt using an adapted internet-based CM procedure (Dallery et al, 2013), allowing for remote biochemical verification of smoking abstinence (see Supplementary Informa- 
tion). Briefly, participants uploaded videos of expired $\mathrm{CO}$ twice per day during a 21-day period (3 days baseline and 18 day quit attempt). Samples meeting criteria for abstinence were reinforced according to an ascending schedule during the 18-day quit attempt. Non-abstinent or missing samples were not reinforced. Of 37 total participants, 14 (37.8\%) maintained continuous abstinence throughout the procedure; the remaining $23(62.2 \%)$ lapsed during the quit attempt $(n=21)$ or failed to achieve more than one consecutive abstinent sample $(n=2)$. Consistent with previous studies and the Society for Research on Nicotine and Tobacco's working group definition of a slip (Hughes et al, 2003; Janes et al, 2010), lapse was defined as any smoking after achieving an initial 24-h abstinence (ie, two consecutive abstinent samples), determined by self-report and/or submission of a CO sample above the required cutoff for abstinence. Smokers who lapsed smoked on an average of $45.6 \%$ of the days during the quit attempt. Subsequent analyses were aimed at differentiating those who lapsed or failed to initiate abstinence (lapse) from those maintaining continuous abstinence (non-lapse).

\section{Resting State Functional Connectivity}

Imaging acquisition and preprocessing. BOLD functional images were acquired using a 3.0 Tesla Siemens Allegra scanner (Erlangen, Germany) with gradient EPI sequence covering 34 interleaved axial slices of $3 \mathrm{~mm}$ thickness with the following parameters: $\mathrm{TR}=2 \mathrm{~s} ; \mathrm{TE}=29 \mathrm{~ms}$; flip angle $=90^{\circ}$; $64 \times 64$ matrix with $\mathrm{FOV}=20 \times 20 \mathrm{~cm}$. A T1-weighted structural image was acquired using a three-dimensional volume MPRAGE pulse sequence covering 176 axial slices of $1 \mathrm{~mm}$ thickness. Images were preprocessed using SPM8 (www.fil.ion.ucl.ac.uk/spm). Images were corrected for slice timing effects and realigned to the mean functional image. Structural images were segmented into native space gray matter and coregistered to the mean of functional images. Functional images were spatially normalized into Montreal Neurologic Institute stereotactic space and resampled to a voxel size of $2 \times 2 \times 2 \mathrm{~mm}$. Images were smoothed with a Gaussian filter set at $6 \mathrm{~mm}$ full-width at half-maximum, and a high-pass filter (128s) was applied. Artifact detection tools implemented in SPM (www.nitrc.org/projects/artifact_detect) were used to identify and adjust for image artifacts related to intensity spiking and motion (see Supplementary Information).

Data analysis. Band-pass filtering $(0.008-0.09 \mathrm{~Hz})$ was applied to preprocessed resting-state images and physiological and movement confounds were removed using a component-based noise correction method implemented in CONN: Functional connectivity toolbox (www.nitrc.org/ projects/conn) (Whitfield-Gabrieli and Nieto-Castanon, 2012). For each subject and session, Fisher Z-transformed image maps were then created by calculating the correlation coefficient of each voxel with the time-series of seed regions of interest (ROIs). Anatomical striatal seeds were created using previously defined ROIs (Sweitzer et al, 2013), and included bilateral VS, encompassing the anterior portions of the ventral caudate and putamen, as well as anterior globus pallidus (Gianaros et al, 2011), and bilateral DS, defined using the WFU_PickAtlas toolbox (www.ansir.wfubmc.edu) to encompass the head, body, and tail of the caudate (Maldjian et al, 2003) (Figure 1). In addition, to minimize potential effects of movement and individual anatomical differences, analyses were rerun using $5 \mathrm{~mm}$ spherical ROIs centered at $\pm 10,11,-6$ (for right and left VS), and $\pm 11,12$, 10 (for right and left DS). Results were unchanged when using spherical ROIs except where noted.

\section{Hypothesis Testing}

First-level Z maps created for each seed were submitted to second-level random effects ANOVA for group analyses conducted within SPM8. Effects of condition (abstinent $v s$ satiated) and lapse status (lapse $v s$ non-lapse) on rsFC were modeled with a $2 \times 2$ ANOVA, with condition entered as a within-subjects factor and lapse status entered as a betweensubjects factor. Age, sex, cigarettes smoked per day, and session order (ie, abstinent or satiated condition first) were entered as covariates of no interest. Hypothesis testing was conducted across the whole brain, encompassing a volume of $1853928 \mathrm{~mm}^{3}$ or 231741 voxels. Corrections for multiple comparisons were made using a combined cluster size and individual voxel threshold method determined by Monte Carlo simulations implemented in 3dClusterSim (AFNI). A cluster of 212 voxels along with an individual voxel significance level of $p<0.005$ was required for an overall corrected false-positive detection rate of $P<0.01$. For activation clusters exhibiting a significant F-test, planned contrasts were conducted within SPM to determine the direction of effects. For selected clusters (discussed below), the first eigenvariate was extracted for further analysis within SPSS.

\section{RESULTS}

\section{Behavioral Measures}

Demographic and baseline smoking variables for lapsers and abstainers are presented in Table 1. Lapsers did not differ significantly from non-lapsers in terms of age, sex, years daily smoking, or severity of nicotine dependence; lapsers smoked significantly more cigarettes per day than nonlapsers $(t=2.25, \mathrm{df}=35, p<0.05)$.

\section{Overall Patterns of Striatal Connectivity}

The pattern of striatal connectivity throughout the brain was first examined, collapsed across both condition and group. As expected, robust connectivity was observed for both right and left VS and DS seeds across multiple cortical and subcortical regions (Supplementary Figure S1). Patterns of connectivity for each seed region were similar to previous reports (Di Martino et al, 2008; Porter et al, 2015).

\section{Main Effects of Condition}

Ventral striatum. Abstinence (relative to satiation) was associated with reduced functional connectivity between the left VS seed and superior frontal gyrus (Figure 1a and Table 2). Abstinence did not increase connectivity between the left VS and any other region, and connectivity with the right VS was not modulated by condition. 
a
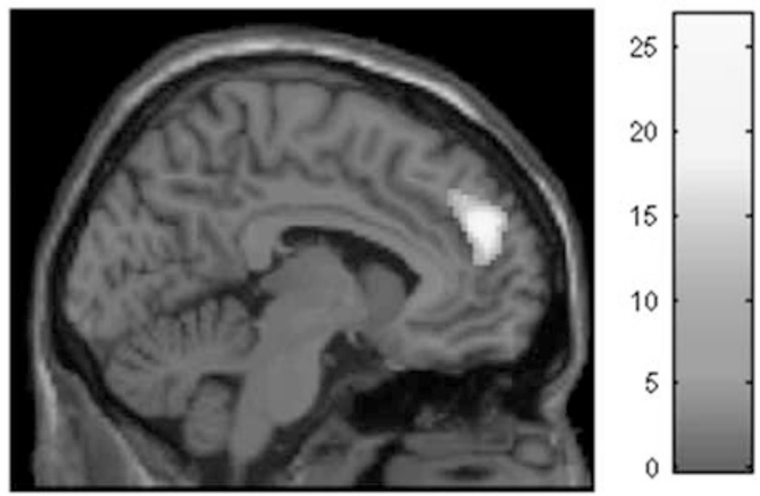

b
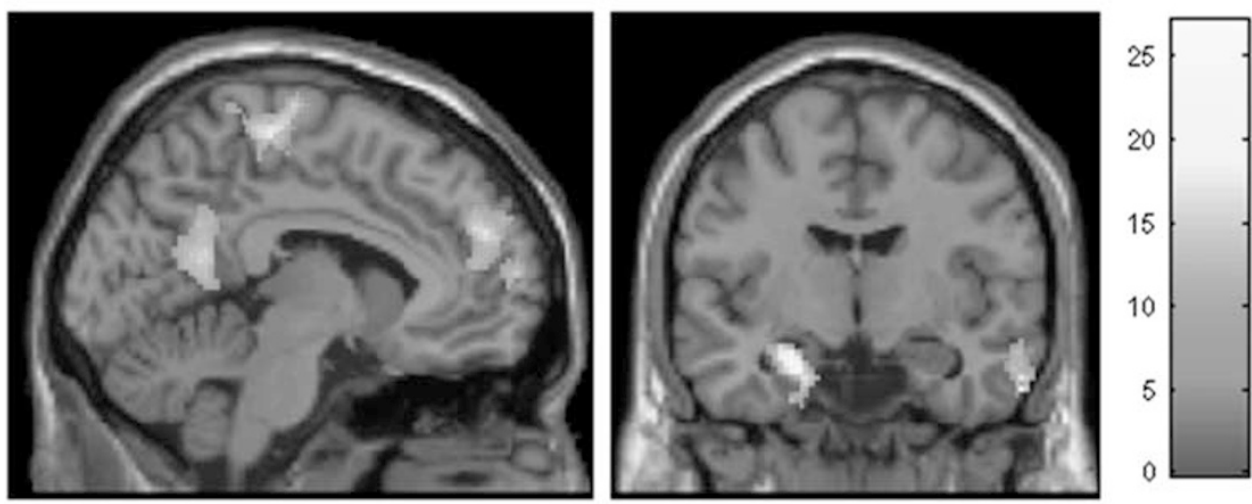

C
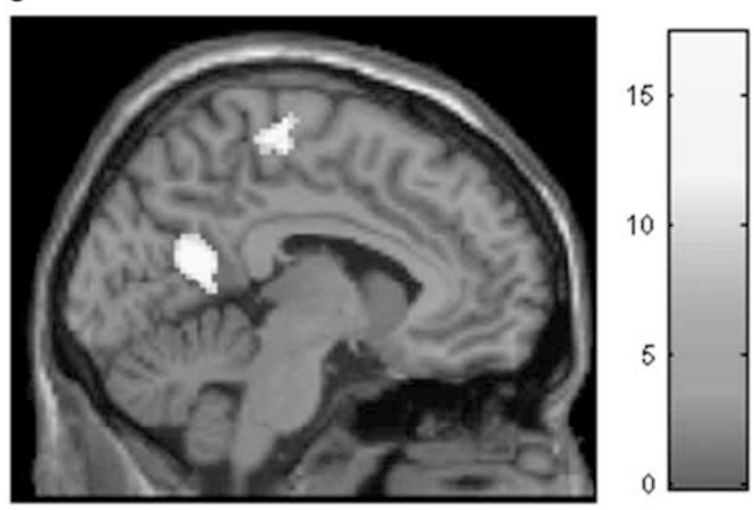

Figure I Main effects of abstinence on functional connectivity with the left ventral striatum (VS) (a), left dorsal striatum (DS) (b) and right DS (c). All activation clusters reflect areas of decreased connectivity with VS and DS seeds during abstinence relative to satiation. Panels are displayed at $x=-4, y=-8$. Colored bars reflect F-values. A full color version of this figure is available at the Neuropsychopharmacology journal online.

Table I Demographic and Baseline Smoking Variables for those Who Lapsed (Lapsers) Versus those Who Maintained Continuous Abstinence (Abstainers) During Contingency Management

\begin{tabular}{|c|c|c|c|c|}
\hline Variable & Lapsers Mean (and SD) $(n=23)$ & Abstainers Mean (and SD) $(n=14)$ & $t$-value $(\mathrm{df}=35)$ & $p$-value \\
\hline Age & $35.3(12.7)$ & $30.9(12.1)$ & $1.04 \mid$ & 0.31 \\
\hline Sex (\% female) & $57 \%$ & $43 \%$ & $0.650^{\mathrm{a}}$ & 0.42 \\
\hline FTND & $3.8(2.6)$ & $3.0(1.9)$ & 1.108 & 0.28 \\
\hline Cigarettes smoked per day & | $5.1(6.5)$ & II.I (4.4) & 2.253 & 0.03 \\
\hline Years daily smoking & $16.2(12.1)$ & $10.5(8.5)$ & 1.526 & 0.14 \\
\hline
\end{tabular}

Abbreviation: FTND, Fagerström Test of Nicotine Dependence.

${ }^{\mathrm{a}}$ Test statistic for sex variable is Pearson $\chi^{2}$. 
Table 2 Areas of Decreased Functional Connectivity with VS and DS During Smoking Abstinence

\begin{tabular}{|c|c|c|c|c|c|}
\hline \multirow[t]{2}{*}{ Region } & \multirow[t]{2}{*}{ Voxels } & \multicolumn{3}{|c|}{ Location } & \multirow[t]{2}{*}{ F-value } \\
\hline & & $x$ & $y$ & $\mathbf{z}$ & \\
\hline \multicolumn{6}{|l|}{ Left VS seed } \\
\hline Superior/medial frontal gyrus & 1079 & -8 & 50 & 26 & 26.87 \\
\hline \multicolumn{6}{|l|}{ Left DS seed } \\
\hline $\begin{array}{l}\text { L hippocampus/ } \\
\text { parahippocampal gyrus }\end{array}$ & 654 & -22 & 0 & -34 & 27.13 \\
\hline R middle temporal gyrus & 464 & 58 & 0 & -20 & 24.81 \\
\hline Supplemental motor area & 1305 & 6 & -22 & 52 & 17.99 \\
\hline Superior/medial frontal gyrus & 1243 & -18 & 56 & 8 & 17.59 \\
\hline Precuneus/posterior cingulate & 547 & -2 & -58 & 14 & 16.56 \\
\hline \multicolumn{6}{|l|}{ Right DS seed } \\
\hline Precuneus & 391 & -2 & -58 & 12 & 17.39 \\
\hline L superior frontal gyrus & 310 & -14 & 42 & 56 & 16.68 \\
\hline $\mathrm{L}$ angular gyrus & 280 & -52 & -68 & 26 & 15.85 \\
\hline Supplemental motor area & 345 & -10 & -22 & 70 & 13.96 \\
\hline
\end{tabular}

Abbreviation: VS, ventral striatum.

Location refers to Montreal Neurological Institute coordinates for peak voxel of each cluster.

Dorsal striatum. For the left DS seed, 24-h abstinence was associated with decreased functional connectivity with the precuneus/posterior cingulate cortex (PCC) and the medial prefrontal cortex (mPFC) - partially overlapping with regions considered part of the default mode network (Figure $1 \mathrm{~b}$ and Table 2). Additionally, abstinence decreased connectivity between the left DS and the left hippocampus and parahippocampal gyrus, supplemental motor area (SMA), and right middle temporal gyrus. A similar but less extensive pattern of decreased connectivity during abstinence relative to satiation was observed for right DS seed (Figure 1c and Table 2). Effects of abstinence for the right DS seed were nonsignificant when using a spherical ROI.

\section{Associations with CM Outcome}

For all seed regions, there were no overall group differences in connectivity between lapsers and non-lapsers. We then examined the condition by lapse outcome interaction to determine whether the magnitude of abstinence-induced changes in connectivity with each seed region differed between lapsers and non-lapsers. Results for each seed region are reported below.

Ventral striatum. A significant condition by group interaction effect was observed for both right and left VS seeds with regions including the bilateral superior temporal gyrus, right insula (including dorsoanterior and posterior regions), dACC and mid-cingulate cortex (MCC), and the right inferior parietal lobule (Figure 2 and Table 3). Additional regions of connectivity exhibiting this interaction pattern were observed for the right VS seed with the left dorsoanterior and posterior insula and SMA, and for the left VS with the right thalamus, putamen, and parahippocampal gyrus, right inferior frontal gyrus, bilateral precuneus, PCC, and bilateral portions of occipital cortex. Followup contrasts within SPM indicated that for all of these areas, non-lapsers exhibited greater connectivity during abstinence relative to satiety, compared with lapsers. In other words, greater abstinence-induced increases in VS connectivity with other corticolimbic regions was associated with better quit outcomes during CM.

Dorsal striatum. For the right DS seed, a significant condition by lapse interaction effect was found for a small cluster in the dACC/MCC (Supplementary Figure S2). Similar to the VS seeds, non-lapsers exhibited greater connectivity during abstinence relative to satiety, compared with lapsers. This effect was nonsignificant with the spherical ROI. There were no significant interaction effects for the left DS.

\section{Follow-Up Analyses}

Pairwise comparisons. Given that significant interaction effects were observed in the right insula and $\mathrm{AACC} / \mathrm{MCC}$ for both right and left VS, and considering the relevance of these regions for addictive disorders (Sutherland et al, 2012), eigenvariates were extracted from clusters in the right insula/ superior temporal gyrus and AACC/MCC for each VS seed (right and left) and plotted to examine the direction of the observed interaction effects. As shown in Figure 2, among non-lapsers, abstinence increased connectivity between the right VS and MCC $(t=3.98, \mathrm{df}=13, p<0.005)$ and right insula ( $t=4.33, \mathrm{df}=13, p<0.001)$; by contrast, lapsers exhibited a trend toward decreased connectivity between right VS and MCC $(t=-1.97, \mathrm{df}=22, p=0.06)$, with no change in the right insula $(t=-1.72, \mathrm{df}=22, p=0.10)$ during abstinence relative to satiation. An identical pattern was observed for connectivity with the left VS, except that the decrease in left VS-MCC connectivity during abstinence among lapsers was significant $(t=-2.12, \mathrm{df}=22, p<0.05)$.

Associations with subjective measures. Using eigenvariates extracted from interaction clusters described above, we next examined whether abstinence-induced changes in rsFC with each VS seed were associated with changes in subjective craving and withdrawal symptoms. As previously reported for this sample, abstinence increased self-reported craving $(t=6.7, \mathrm{df}=36, p<0.001)$ and withdrawal symptoms $(t=3.7, \mathrm{df}=36, p<0.001)$, and the magnitude of abstinenceinduced craving was greater for non-lapsers than for lapsers $(t=2.7, \mathrm{df}=35, p<0.05) \quad$ (Sweitzer et al, 2016). Across individuals, abstinence-induced craving (abstinence minus satiation) was positively correlated with abstinence-induced increase in rsFC between the right VS and right insula $(r=0.43, p<0.01)$ and right VS and MCC $(r=0.42, p<0.01)$. Similar correlations were found between self-reported craving and rsFC with the left VS seed. There were no associations between change in rsFC and change in withdrawal symptoms. 
a
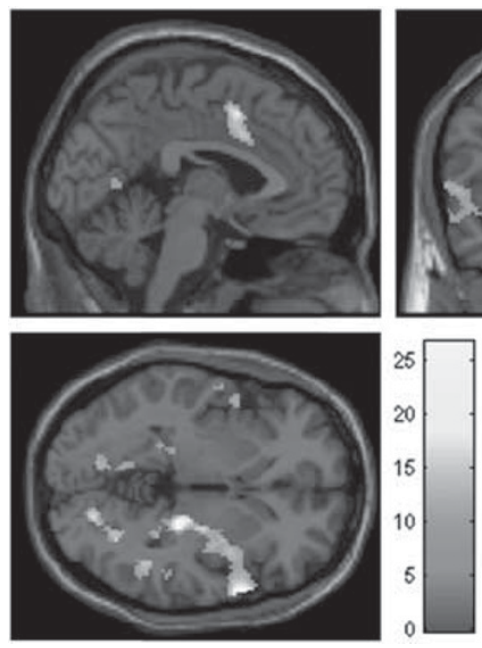

C

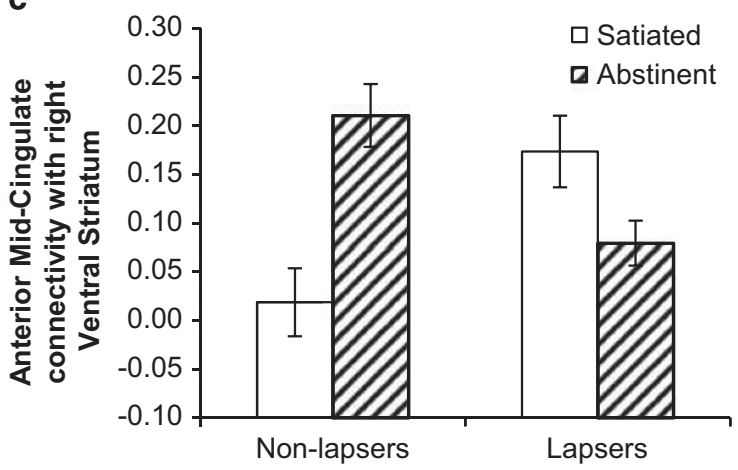

Cessation Outcome b
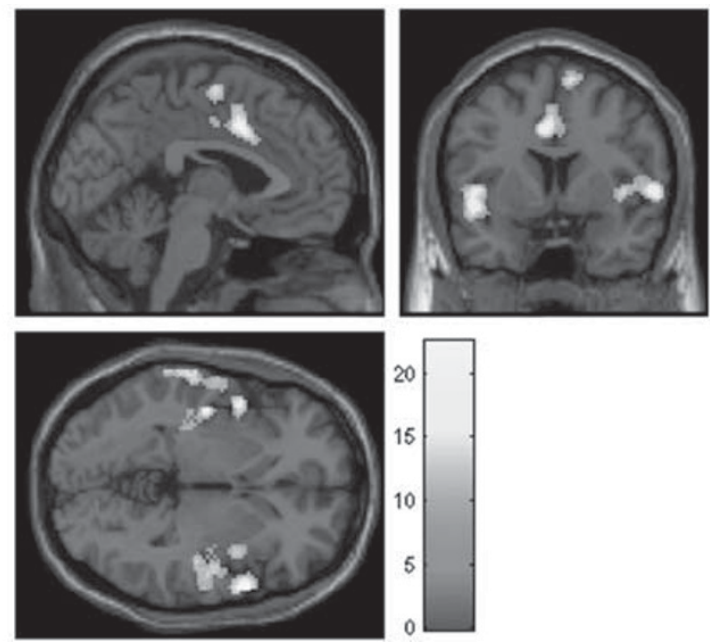

d

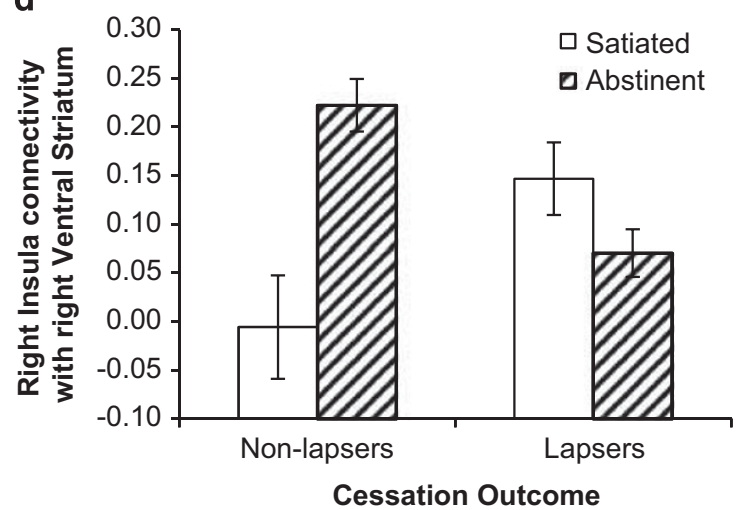

Figure 2 Patterns of functional connectivity with the left (a) and right (b) ventral striatum associated with a significant condition (abstinent vs satiated) by lapse status (lapse vs abstainers) interaction. Colored bars represent F-values. Panels are displayed at $x=-2, y=6, z=-2$. (c, d). Connectivity with the right ventral striatum plotted by condition and lapse status, based on eigenvariate extracted from functional clusters in the mid-cingulate cortex (c) and right insula/ superior temporal gyrus (d). Error bars reflect standard error of the mean. A full color version of this figure is available at the Neuropsychopharmacology journal online.

Connectivity analyses with functional ROIs. To examine the specificity of interaction effects to the striatum, additional confirmatory analyses were performed using MCC and right insula seeds functionally defined based on preceding connectivity analyses. Seeds for these regions included voxels showing a significant condition by group interaction with both right and left VS seeds, and whole-brain analyses were repeated to determine the involvement of any circuits beyond the 'reverse' pathways connecting back to the striatum. As shown in Supplementary Figure S3, the expected condition by group interaction effect was found for both cingulate and insula seeds in the bilateral striatum encompassing bilateral caudate and putamen and extending to ventromedial prefrontal cortex, and for the MCC, to a small portion of the brainstem. No interaction effects were observed for connectivity between the insula and MCC, supporting the specificity of these findings to connectivity with the striatum.

\section{DISCUSSION}

We used a seed-based approach to examine changes in rsFC with the ventral and dorsal striatum as a function of 24-h abstinence from smoking (relative to satiation), and whether magnitude of pre-quit changes differed between lapsers vs non-lapsers during a quit attempt supported by CM. We observed robust abstinence-induced increases in connectivity with right and left VS across a network of regions previously implicated in addictive disorders, including the insula, superior temporal gyrus, and anterior/mid-cingulate cortex; however, this pattern was only observed among smokers who maintained continuous abstinence during CM, while no effect or the opposite pattern was observed for those who lapsed. To our knowledge, this is the first demonstration of differential changes in rsFC during 24-h abstinence associated with quit outcomes. These findings suggest that abstinence-induced increases in VS connectivity with cortical regions implicated in cognitive and behavioral control may be a marker for better cessation outcomes.

The anterior MCC/dACC and insular regions that exhibited increased striatal connectivity during abstinence among non-lapsers have been proposed to underlie a 'core' task-set system involved in goal-directed cognition and intentional motor control (Dosenbach et al, 2006; Hoffstaedter et al, 2013). Activity in these regions is thought to reflect monitoring of the external environment and 
Table 3 Areas Exhibiting a Significant Condition (Abstinent Vs Satiated) by Lapse Status (Lapsers vs Abstainers) Interaction for Functional Connectivity with the Left and Right VS

\begin{tabular}{|c|c|c|c|c|c|}
\hline \multirow[t]{2}{*}{ Region } & \multirow[t]{2}{*}{ Voxels } & \multicolumn{3}{|c|}{ Location } & \multirow[t]{2}{*}{ F-value } \\
\hline & & $x$ & $y$ & $\mathbf{z}$ & \\
\hline \multicolumn{6}{|l|}{ Left VS seed } \\
\hline $\begin{array}{l}\text { R superior/middle temporal } \\
\text { gyrus/insula/putamen/ } \\
\text { hippocampus/thalamus }\end{array}$ & 1274 & 62 & 12 & 0 & 26.64 \\
\hline $\begin{array}{l}\text { Mid-cingulate/dorsal anterior } \\
\text { cingulated }\end{array}$ & 643 & -10 & 8 & 38 & 24.26 \\
\hline $\begin{array}{l}\mathrm{R} \text { inferior parietal lobule/ } \\
\text { precuneus }\end{array}$ & 2369 & 60 & -38 & 22 & 22.24 \\
\hline $\mathrm{R}$ middle/inferior frontal gyrus & 513 & 54 & 6 & 46 & 21.36 \\
\hline $\mathrm{L}$ precuneus & 226 & -12 & -86 & 44 & 21.10 \\
\hline L superior/middle temporal gyrus & 666 & -52 & -8 & -10 & 18.68 \\
\hline R occipital lobe & 306 & 18 & -78 & -4 & 17.59 \\
\hline L occipital lobe & 411 & -14 & -60 & 8 & 16.00 \\
\hline $\mathrm{R}$ posterior cingulate & 218 & 18 & -54 & 12 & 14.94 \\
\hline \multicolumn{6}{|l|}{ Right VS seed } \\
\hline $\begin{array}{l}\text { R superior/middle temporal } \\
\text { gyrus/insula }\end{array}$ & 918 & 60 & 10 & 2 & 22.53 \\
\hline $\begin{array}{l}\text { Mid-cingulate/dorsal anterior } \\
\text { cingulate }\end{array}$ & 634 & 8 & 20 & 16 & 21.08 \\
\hline $\begin{array}{l}\text { L superior/middle temporal } \\
\text { gyrus/insula }\end{array}$ & 835 & -50 & 2 & -12 & 20.96 \\
\hline $\mathrm{R}$ inferior parietal lobule & 372 & 58 & -34 & 30 & 20.75 \\
\hline Supplemental motor area & 451 & 12 & 2 & 68 & 17.49 \\
\hline
\end{tabular}

Abbreviations: DS, dorsal striatum; VS, ventral striatum.

Location refers to Montreal Neurological Institute coordinates for peak voxel of each cluster.

internal subjective states, and is integrally linked with processes of response selection and action preparation (Taylor et al, 2009). The insula is thought to play a central role in interoceptive awareness of internal, subjective states, including craving to smoke (Kuhn and Gallinat, 2011; Naqvi and Bechara, 2009; Wang et al, 2014); and insula lesions have been associated with abrupt disruption of tobacco addiction (Naqvi et al, 2007). The anterior MCC, through its interconnections with prefrontal cortical regions, striatum, insula, and SMA, is thought to play a central role in cognitive control and has been proposed as a key interface integrating affective state, reinforcement learning, and goal anticipation to guide planned behavior (Shackman et al, 2011). Taken together, stronger intrinsic VS connectivity with this network may reflect both an enhanced awareness of subjective urge to smoke and a greater ability to regulate behavior in the service of goals for maintaining abstinence. Although speculative, this interpretation is consistent with the findings that increased connectivity between the VS and cingulo-insular network during abstinence was associated with increased craving, but also better quit outcomes. However, it is important to consider that higher abstinence-induced craving was itself associated with better quit outcomes (Sweitzer et al, 2016). As such, it is unknown how these findings might differ under conditions in which higher levels of craving are associated with higher likelihood of lapse, as has been observed in prior studies.

If increased VS connectivity with the cingulo-insular network reflects a stronger degree of cognitive control over motivationally relevant stimuli, thereby enhancing the ability to inhibit the impulse to smoke, then it is somewhat surprising that such regulatory pathways were increased during abstinence among non-lapsers, particularly as tobacco withdrawal is associated with diminished ability to resist smoking (Roche et al, 2014). However, these results are consistent with previous studies demonstrating that connectivity within this network is strengthened during smoking withdrawal (Cole et al, 2010; Huang et al, 2014; Sutherland et al, 2013); but also that connectivity between similar regions is attenuated among smokers compared with nonsmokers (Hong et al, 2010), and is negatively correlated with nicotine dependence (Hong et al, 2009) and likelihood of relapse (Addicott et al, 2015; Janes et al, 2010). Thus, enhanced connectivity across regions including the insula, cingulate, and striatum appears to be protective when assessed as a trait-level marker independent of abstinence state, whereas state-based increases in connectivity strength co-occur with increases in subjective craving and withdrawal (Huang et al, 2014; Sutherland et al, 2013), similar to that observed here. The present findings build upon this prior research to highlight the importance of individual differences in the effects of abstinence on connectivity strength and suggest that withdrawal-induced modulation of connectivity may be associated with better cessation outcomes.

To our knowledge, only two published studies have examined intrinsic connectivity strength in association with smoking cessation outcomes. Janes et al (2010) used independent component analysis to examine differences in task-based connectivity among women who lapsed compared with non-lapsers during an 8-week quit attempt. In that study, women who lapsed exhibited weaker connectivity between a network containing the insula and cognitive control regions of the $\mathrm{dACC}$ and dorsolateral prefrontal cortex than those who maintained abstinence. Similarly, in another larger study, smokers who relapsed during a 3-month quit attempt exhibited weaker rsFC between a posterior insula seed region and portions of the putamen and somatosensory cortex (Addicott et al, 2015). In contrast to these findings, no main effect of lapse outcome was observed in the present study, and the condition by lapse interaction appeared to be driven, in part, by lower VS connectivity during satiation - the condition most closely paralleling prior studies. One possible explanation for this discrepancy is that smokers in both aforementioned studies were administered nicotine replacement during the quit attempts. As such, connectivity assessed during a satiated state may have more closely mimicked the condition of continued transdermal nicotine administration during smoking cessation, particularly as nicotine replacement has been shown to modulate network connectivity in key regions (Cole et al, 2010; Sutherland et al, 2012). By contrast, functional connectivity following 24-h abstinence in the present study may be more strongly associated with smoking behavior during an unmedicated quit attempt.

The present study also examined changes in DS connectivity as a function of abstinence condition and lapse 
outcome. Across subjects and conditions, overall patterns of connectivity with VS and DS seeds were overlapping but distinct and conformed to those previously described in the literature (Di Martino et al, 2008; Porter et al, 2015), with greater VS connectivity observed with limbic regions involved in affective processing (eg, insula and cingulate cortex), and greater DS connectivity with regions involved in executive functioning (eg, dorsolateral prefrontal cortex). In contrast to the VS interaction effects, the primary finding for the DS seeds was a main effect of decreased connectivity during abstinence relative to satiation with the MPFC, PCC, hippocampus, and SMA, particularly for the left DS. While the VS is involved in incentive salience and reward valuation and plays a central role in early stages of drug acquisition, the transition to compulsive drug use is thought to be mediated by a shift in dopaminergic innervation from ventral to dorsal striatum (Everitt and Robbins, 2005; Pierce and Vanderschuren, 2010). The mPFC, PCC, and hippocampus are central components of the 'default mode network' thought to reflect self-referential processing in the absence of explicit task demands (Buckner et al, 2008; Raichle et al, 2001). Inefficient suppression of the default mode network by task-positive regions has been associated with a range of negative clinical outcomes, and is worsened by withdrawal from smoking (Lerman et al, 2014). That abstinence decreased DS connectivity with these regions is somewhat surprising, given that increased connectivity with these areas (and with SMA) could underlie an increased ruminative preoccupation with smoking-related urges and preparation for habitual smoking behavior (Sutherland et al, 2012). An alternative interpretation is that decreased DS connectivity with these regions during withdrawal reflects a disengagement of habitual, automated, and largely unconscious processes underlying maintenance of smoking behavior, in favor of the conscious perception of craving emerging with the disruption of compulsive behavior (Tiffany and Conklin, 2000).

The present study has several strengths, including prospective design and examination of rsFC during both satiated and abstinent conditions. An important limitation is that resting state measures were conducted after a rewarded guessing task eliciting striatal activation to both smoking and monetary rewards (Sweitzer et al, 2013, 2016). Given that cognitive task performance can influence subsequent measures of rsFC (Albert et al, 2009), it is unknown to what extent group differences and abstinence effects on rsFC were due to residual effects of reactivity during the reward task. However, it is important to note that task-related effects were stimulus-specific (ie, dependent on reward type within the task) and did not reflect overall differences in engagement of reward-related regions. The results are also limited by a relatively small sample size, particularly among the non-lapse group, recruitment of only Caucasian smokers, relatively low level of dependence within the sample potentially limiting generalizability to heavier, more dependent smokers, and short duration of follow-up. Small sample size, in particular, could have contributed to low power and type II error when examining between-group differences, and can lead to an inflated estimate of effect size (see Button et al, 2013); as such, it will be critical for future results to provide replication in a larger, more representative sample. In addition, the relatively high abstinence rates (38\%) observed at the conclusion of the 3-week quit period may not be predictive of longer-term outcomes, and our assessment of nontreatment seekers participating in CM may not generalize to smokers attempting to quit by other means. Future studies should examine the replicability of these findings in a larger sample of treatment-seeking smokers quitting over a longer period of time. A final caveat is that the seed-based approach used here was optimal for targeting connectivity with specific striatal ROIs, but did not allow for examination of internetwork correlations. Despite these limitations, the present findings have important implications for understanding mechanisms contributing to vulnerability and suggest that interventions to enhance striatal connectivity during abstinence, particularly among those at greatest risk, may help reduce risk of lapse.

\section{FUNDING AND DISCLOSURE}

This research was supported by NIH grant (DA027441) to ECD. MMS was supported by NSF IGERT award (0549352) and the Center for the Neural Basis of Cognition. The authors declare no conflict of interest.

\section{REFERENCES}

Addicott MA, Sweitzer MM, Froeliger B, Rose JE, McClernon FJ (2015). Increased functional connectivity in an insula-based network is associated with improved smoking cessation outcomes. Neuropsychopharmacology 40: 2648-2656.

Albert NB, Robertson EM, Miall RC (2009). The resting human brain and motor learning. Curr Biol 19: 1023-1027.

Buckner RL, Andrews-Hanna JR, Schacter DL (2008). The brain's default network: anatomy, function, and relevance to disease. Ann NY Acad Sci 1124: 1-38.

Buhler M, Vollstadt-Klein S, Kobiella A, Budde H, Reed LJ, Braus DF et al (2010). Nicotine dependence is characterized by disordered reward processing in a network driving motivation. Biol Psychiatry 67: 745-752.

Button KS, Ioannidis JP, Mokrysz C, Nosek BA, Flint J, Robinson ES et al (2013). Power failure: why small sample size undermines the reliability of neuroscience. Nat Rev Neurosci 14: 365-376.

Cole DM, Beckmann CF, Long CJ, Matthews PM, Durcan MJ, Beaver JD (2010). Nicotine replacement in abstinent smokers improves cognitive withdrawal symptoms with modulation of resting brain network dynamics. NeuroImage 52: 590-599.

Dallery J, Raiff BR, Grabinski MJ (2013). Internet-based contingency management to promote smoking cessation: a randomized controlled study. J Appl Behav Anal 46: 750-764.

David SP, Munafo MR, Johansen-Berg H, Smith SM, Rogers RD, Matthews PM et al (2005). Ventral striatum/nucleus accumbens activation to smoking-related pictorial cues in smokers and nonsmokers: a functional magnetic resonance imaging study. Biol Psychiatry 58: 488-494.

Di Martino A, Scheres A, Margulies DS, Kelly AM, Uddin LQ, Shehzad Z et al (2008). Functional connectivity of human striatum: a resting state FMRI study. Cereb Cortex 18: 2735-2747.

Dosenbach NU, Visscher KM, Palmer ED, Miezin FM, Wenger KK, Kang HC et al (2006). A core system for the implementation of task sets. Neuron 50: 799-812.

Engelmann JM, Versace F, Robinson JD, Minnix JA, Lam CY, Cui Y et al (2012). Neural substrates of smoking cue reactivity: a metaanalysis of fMRI studies. NeuroImage 60: 252-262. 
Everitt BJ, Robbins TW (2005). Neural systems of reinforcement for drug addiction: from actions to habits to compulsion. Nat Neurosci 8: 1481-1489.

Gianaros PJ, Manuck SB, Sheu LK, Kuan DC, Votruba-Drzal E, Craig AE et al (2011). Parental education predicts corticostriatal functionality in adulthood. Cereb Cortex 21: 896-910.

Heatherton TF, Kozlowski LT, Frecker RC, Fagerstrom KO (1991). The Fagerstrom Test for Nicotine Dependence: a revision of the Fagerstrom Tolerance Questionnaire. Br J Addict 86: 1119-1127.

Hoffstaedter F, Grefkes C, Zilles K, Eickhoff SB (2013). The 'what' and 'when' of self-initiated movements. Cereb Cortex 23: 520-530.

Hong LE, Gu H, Yang Y, Ross TJ, Salmeron BJ, Buchholz B et al (2009). Association of nicotine addiction and nicotine's actions with separate cingulate cortex functional circuits. Arch Gen Psychiatry 66: 431-441.

Hong LE, Hodgkinson CA, Yang Y, Sampath H, Ross TJ, Buchholz B et al (2010). A genetically modulated, intrinsic cingulate circuit supports human nicotine addiction. Proc Natl Acad Sci USA 107: 13509-13514.

Huang W, King JA, Ursprung WW, Zheng S, Zhang N, Kennedy DN et al (2014). The development and expression of physical nicotine dependence corresponds to structural and functional alterations in the anterior cingulate-precuneus pathway. Brain Behav 4: 408-417.

Hughes JR (2007). Effects of abstinence from tobacco: valid symptoms and time course. Nicotine Tob Res 9: 315-327.

Hughes JR, Hatsukami D (1986). Signs and symptoms of tobacco withdrawal. Arch Gen Psychiatry 43: 289-294.

Hughes JR, Shiffman S, Callas P, Zhang J (2003). A meta-analysis of the efficacy of over-the-counter nicotine replacement. Tob Control 12: 21-27.

Janes AC, Nickerson LD, Frederick Bde B, Kaufman MJ (2012). Prefrontal and limbic resting state brain network functional connectivity differs between nicotine-dependent smokers and non-smoking controls. Drug Alcohol Depend 125: 252-259.

Janes AC, Pizzagalli DA, Richardt S, de BFB, Chuzi S, Pachas G et al (2010). Brain reactivity to smoking cues prior to smoking cessation predicts ability to maintain tobacco abstinence. Biol Psychiatry 67: 722-729.

Kuhn S, Gallinat J (2011). Common biology of craving across legal and illegal drugs-a quantitative meta-analysis of cue-reactivity brain response. Eur J Neurosci 33: 1318-1326.

Lerman C, Gu H, Loughead J, Ruparel K, Yang Y, Stein EA (2014). Large-scale brain network coupling predicts acute nicotine abstinence effects on craving and cognitive function. JAMA Psychiatry 71: 523-530.

Maldjian JA, Laurienti PJ, Kraft RA, Burdette JH (2003). An automated method for neuroanatomic and cytoarchitectonic atlasbased interrogation of fMRI data sets. NeuroImage 19: 1233-1239.

McClernon FJ, Kozink RV, Lutz AM, Rose JE (2009). 24-h smoking abstinence potentiates $\mathrm{MMRI}-\mathrm{BOLD}$ activation to smoking cues in cerebral cortex and dorsal striatum. Psychopharmacology (Berl) 204: 25-35.

Middleton FA, Strick PL (2002). Basal-ganglia 'projections' to the prefrontal cortex of the primate. Cereb Cortex 12: 926-935.

Naqvi NH, Bechara A (2009). The hidden island of addiction: the insula. Trends Neurosci 32: 56-67.

Naqvi NH, Rudrauf D, Damasio H, Bechara A (2007). Damage to the insula disrupts addiction to cigarette smoking. Science 315: 531-534.

Nestler EJ (2004). Molecular mechanisms of drug addiction. Neuropharmacology 47 (Suppl 1): 24-32.

Pierce RC, Vanderschuren LJ (2010). Kicking the habit: the neural basis of ingrained behaviors in cocaine addiction. Neurosci Biobehav Rev 35: 212-219.
Porter JN, Roy AK, Benson B, Carlisi C, Collins PF, Leibenluft E et al (2015). Age-related changes in the intrinsic functional connectivity of the human ventral vs. dorsal striatum from childhood to middle age. Dev Cogn Neurosci 11: 83-95.

Raichle ME, MacLeod AM, Snyder AZ, Powers WJ, Gusnard DA, Shulman GL (2001). A default mode of brain function. Proc Natl Acad Sci USA 98: 676-682.

Reynolds SM, Zahm DS (2005). Specificity in the projections of prefrontal and insular cortex to ventral striatopallidum and the extended amygdala. J Neurosci 25: 11757-11767.

Roche DJ, Bujarski S, Moallem NR, Guzman I, Shapiro JR, Ray LA (2014). Predictors of smoking lapse in a human laboratory paradigm. Psychopharmacology (Berl) 231: 2889-2897.

Shackman AJ, Salomons TV, Slagter HA, Fox AS, Winter JJ, Davidson RJ (2011). The integration of negative affect, pain and cognitive control in the cingulate cortex. Nat Rev Neurosci 12: 154-167.

Sutherland MT, Carroll AJ, Salmeron BJ, Ross TJ, Hong LE, Stein EA (2013). Down-regulation of amygdala and insula functional circuits by varenicline and nicotine in abstinent cigarette smokers. Biol Psychiatry 74: 538-546.

Sutherland MT, McHugh MJ, Pariyadath V, Stein EA (2012). Resting state functional connectivity in addiction: lessons learned and a road ahead. NeuroImage 62: 2281-2295.

Sweitzer MM, Geier CF, Denlinger R, Forbes EE, Raiff BR, Dallery J et al (2016). Blunted striatal response to monetary reward anticipation during smoking abstinence predicts lapse during a contingency-managed quit attempt. Psychopharmacology (Berl) 233: 751-760.

Sweitzer MM, Geier CF, Joel DL, McGurrin P, Denlinger RL, Forbes EE et al (2013). Dissociated effects of anticipating smoking versus monetary reward in the caudate as a function of smoking abstinence. Biol Psychiatry 76: 681-688.

Taylor KS, Seminowicz DA, Davis KD (2009). Two systems of resting state connectivity between the insula and cingulate cortex. Hum Brain Mapp 30: 2731-2745.

Tiffany ST, Conklin CA (2000). A cognitive processing model of alcohol craving and compulsive alcohol use. Addiction 95 (Suppl 2): S145-S153.

Toll BA, Katulak NA, McKee SA (2006). Investigating the factor structure of the Questionnaire on Smoking Urges-Brief (QSU-Brief). AddictBehav 31: 1231-1239.

Versace F, Engelmann JM, Robinson JD, Jackson EF, Green CE, Lam CY et al (2014). Prequit fMRI responses to pleasant cues and cigarette-related cues predict smoking cessation outcome. Nicotine Tob Res 16: 697-708.

Volkow ND, Fowler JS, Wang GJ, Baler R, Telang F (2009). Imaging dopamine's role in drug abuse and addiction. Neuropharmacology 56 (Suppl 1): 3-8.

Wang K, Yang J, Zhang S, Wei D, Hao X, Tu S et al (2014). The neural mechanisms underlying the acute effect of cigarette smoking on chronic smokers. PLoS One 9: e102828.

Whitfield-Gabrieli S, Nieto-Castanon A (2012). Conn: a functional connectivity toolbox for correlated and anticorrelated brain networks. Brain Connect 2: 125-141.

Wilson SJ, Delgado MR, McKee SA, Grigson PS, MacLean RR, Nichols TT et al (2014). Weak ventral striatal responses to monetary outcomes predict an unwillingness to resist cigarette smoking. Cogn Affect Behav Neurosci 14: 1196-1207.

Wright CI, Groenewegen HJ (1996). Patterns of overlap and segregation between insular cortical, intermediodorsal thalamic and basal amygdaloid afferents in the nucleus accumbens of the rat. Neuroscience 73: 359-373.

Supplementary Information accompanies the paper on the Neuropsychopharmacology website (http://www.nature.com/npp) 